\title{
Undetected and underserved: the untold story of patients who had a minor stroke
}

\author{
Equity of access is particularly concerning for minor stroke
}

$\mathrm{M}$ edical advances, such as stroke units, improved primary and secondary stroke prevention, and hyperacute treatments have revolutionised acute stroke management. ${ }^{1}$ The lessening of stroke severity as a result of such ground-breaking initiatives has, however, led to a larger proportion of individuals returning to community living following minor strokes ${ }^{2}$ (ie, with minimal motor deficits or no obvious sensory abnormality). In this article, we review current literature to identify the potential difficulties experienced following a minor stroke.

Individuals who survive a more severe stroke often undergo extensive multidisciplinary rehabilitation in an inpatient setting. By contrast, patients who have a minor stroke are likely to be discharged home early, often with limited referrals to services beyond their general practitioner. ${ }^{3}$ This is despite increasing evidence that survivors of minor stroke may have persisting strokerelated impairments that require rehabilitation. ${ }^{4}$ These "hidden" impairments may not become apparent until after discharge, when the patient attempts to resume their usual daily activities. ${ }^{2,4}$ Edwards and colleagues ${ }^{4}$ found that despite full independence with personal activities of daily living, $87 \%$ of patients who had a minor stroke reported residual difficulties with mobility, concentration, and participation in social activities and physically demanding leisure activities such as golf. These persisting subtle impairments may cause social and economic disruption for the individual and their family; however, due to difficulties identifying them in the hospital setting, it may result in poor coordination between primary and secondary care, especially if the patient is deemed fully independent at discharge. When the impairments are

Emma C Finch, ${ }^{1,2}$

Michele M Foster ${ }^{3}$ Jennifer Fleming ${ }^{1,2}$

Philip D Aitken ${ }^{2,4}$

Ian Williams ${ }^{4}$

Tegan Cruwys

Linda Worrall

1 University of

Queensland

Brisbane, QLD.

2 Princess Alexandra Hospital, Brisbane, QLD.

3 Menzies Health Institute Queensland

Griffith University,

Brisbane, QLD.

4 Camp Hill Healthcare,

Brisbane, QLD.

e.finch@uq.edu.au

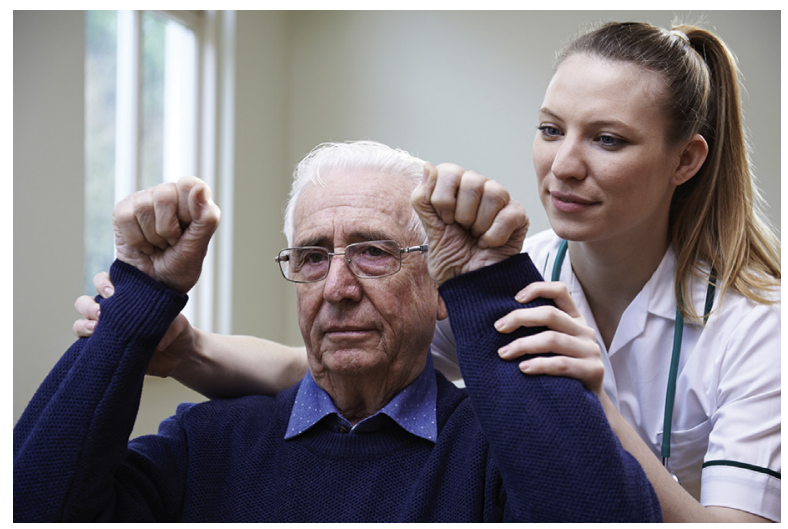

quality of life. ${ }^{2}$ Studies have shown that between oneand two-thirds of minor stroke survivors have compromised social participation outcomes. ${ }^{2,4}$ Edwards and colleagues ${ }^{4}$ found that $62 \%$ of patients who had a mild stroke had difficulty returning to employment or volunteer work, while $36 \%$ had reduced social activity 6 months after the stroke. Since about $30 \%$ of strokes occur in individuals under 65 years of age, ${ }^{8}$ these figures are particularly troubling. It is worth noting, however, that participants in the study by Edwards and colleagues ${ }^{4}$ had experienced a single ischaemic stroke and had a mean age of 64.74 years (range $=20-97$ years). Therefore, as about only half of the participants ${ }^{4}$ in the study fell into the young stroke category, it is unknown how accurately these figures reflect the return to work status specifically of younger patients who had a minor stroke.

The 2014 National Stroke Foundation Rehabilitation audit ${ }^{9}$ found that less than $40 \%$ of patients who had a stroke received a psychological assessment before discharge. Formal neuropsychological assessment is expensive and not available in many areas and so inpatients rarely receive one, even if experiencing obvious impairments, such as aphasia or pronounced memory deficits. For people who have had a minor stroke, impairments are even less obvious and may manifest as a diverse range of milder cognitive problems, including attentional neglect or reduced processing speed. A neuropsychological assessment could identify these deficits and their impact on functioning and make recommendations for compensatory strategies or adjustments to reduce this impact.

Mental health problems, in particular depression, are prevalent regardless of stroke severity, with $25-29 \%$ of patients who have had a minor stroke reporting depression in the first year. ${ }^{10,11}$ Early and late onset poststroke depression has been associated with disability and poor physical and mental health at 1 year, ${ }^{11}$ and with a 
reduced likelihood of driving a vehicle, participating in sports or recreational activities and interpersonal relationships at 6 months after the stroke. ${ }^{12}$ It is encouraging that improvement of depression within the first year after the stroke has been associated with better functional outcomes and quality of life. ${ }^{10}$ This highlights the need to regularly monitor patients after a minor stroke to identify and treat depression as soon as possible.

Despite apparent good recovery, depression is a risk and some patients require referral to services, medication and psychological support in a coordinated manner.

As with most patients who have had a stroke, patients who have had a minor stroke are usually unable to drive for a period of time, relying instead on public transport, family members or unapproved driving for transport to medical appointments and other destinations. Research has found that one in four young survivors of stroke (aged 18-65 years) return to driving within 1 month after the stroke, despite recommendations to the contrary. ${ }^{13}$ Drivers who have had a minor stroke perform significantly worse on complex tasks, with greater cognitive load (eg, turning across oncoming traffic and bus following), and make twice the number of driving errors compared with control subjects. ${ }^{14}$ In addition to the detrimental influence of spatial, visual and cognitive impairments, the risk of seizure contributes to the moratorium on driving after a stroke. Premature return to driving may reflect poor compliance with advice, which is perceived as inconvenient and perhaps not fully explained to patients. Providing patients who have had a minor stroke with education about driving restrictions and alternative transport options and ongoing monitoring of driving fitness should be part of primary health care.

Patients who have had a minor stroke are also at risk of hospital re-admissions due to other medical conditions. For example, patients who have had a minor stroke have a heightened risk of experiencing a subsequent cardiovascular event. ${ }^{15}$ They may also have an array of concomitant medical conditions, including diabetes mellitus, atrial fibrillation and congestive cardiac failure, ${ }^{15}$ and may benefit from a coordinated approach to manage these comorbidities and prevent hospital re-admission.

Six months after a minor stroke, patients do significantly less high intensity physical activity compared with the activity done before the stroke, and despite the benefits of physical activity for future stroke prevention, they tend not to take up new high intensity activities. ${ }^{12}$ Indeed, Kono and colleagues ${ }^{16}$ found that higher levels of exercise in the form of daily step counts were associated with a reduced risk of new vascular events following minor stroke. Patients who have had a minor stroke and are living in the community may benefit from education about secondary stroke prevention. A GP-led multifaceted and target-based approach to secondary stroke prevention may be ideal for this population, especially given that a combination of medications (eg, aspirin, a statin and an antihypertensive agent), exercise and dietary modifications have been found to produce a cumulative relative risk reduction of stroke by $80 \%{ }^{17}$

\section{Conclusion}

In summary, minor stroke is a chronic health condition with long term impairment and disability. ${ }^{2}$ Residual impairments and comorbidities often require the involvement of multiple health care providers, the need for which may not always be evident at the time of stroke. Community-living patients who had a minor stroke may currently be managed through initiatives such as the CDM program. Access to CDM items can be problematic and, due to the mild nature of minor stroke, it is likely that these items will be overlooked. The five sessions per calendar year under the CDM program - which include a range of allied health services, such as speech pathology, occupational therapy, psychology and physiotherapy, with a Medicare rebate that may cover the total cost depending on whether the provider accepts the Medicare benefit as full payment for the service - are often inadequate for patients who have a more complex situation, but may be ideal in the population who have had a minor stroke and hence, a good use of existing resources. Therefore, we need to audit existing strategies in primary care to uncover which processes are working well, and which require attention. This is particularly pertinent given the creation of new government initiatives, including the National Disability Insurance Scheme (in which, however, patients who have had a minor stroke look unlikely to be eligible), and Primary Health Networks within the Health Care Home framework.

A GP-led approach that coordinates a range of primary and allied health professionals close to the home of patients who have had a minor stroke may be the ideal way to meet the needs of this population and prevent costly re-admissions to hospital, while simultaneously maximising quality of life. To ensure that communitydwelling patients who have had a minor stroke and have unmet needs are not missed, we need a coordinated, integrated primary health care response that detects and manages impairments and activity restrictions as they arise, along with medical comorbidity management and self-management support. At a minimum, we need to ensure that all patients who have had a minor stroke, regardless of their geographic location, have improved access to formal neuropsychological assessment, falls prevention, exercise programs and more extensive Medicare-based allied health funding if required. The key to this is auditing existing programs and investigating the relevance of new government initiatives as they arise for these patients, while also improving the communication between hospitals and primary health care services. Further study of the unmet needs and mechanisms for ensuring access for all patients who have had a stroke is also vital.

Competing interests: Emma Finch, Michele Foster, Jennifer Fleming, Philip Aitken, lan Williams, Tegan Cruwys and Linda Worrall have received a University of Queensland Collaborative Industry and Engagement Grant for a project investigating patient outcomes following minor stroke.

Provenance: Not commissioned; externally peer reviewed.

(c) 2017 AMPCo Pty Ltd. Produced with Elsevier B.V. All rights reserved.

References are available online at www.mja.com.au. 
1 National Stroke Foundation. National Stroke Audit - Acute services report 2015. Melbourne: National Stroke Foundation; 2015. https://informme.org. au/stroke-data/Acute-audits (accessed Oct 2016).

2 Tellier M, Rochette A. Falling through the cracks: a literature review to understand the reality of mild stroke survivors. Top Stroke Rehabil 2009; 16: 454-462.

3 Fride Y, Adamit T, Maeir A, et al. What are the correlates of cognition and participation to return to work after first ever mild stroke? Top Stroke Rehabil 2015; 22: 317-325.

4 Edwards DF, Hahn M, Baum C, Dromerick AW. The impact of mild stroke on meaningful activity and life satisfaction. J Stroke Cerebrovasc Dis 2006; 15: 151-157.

5 Simmonds F, Dawber J, Green JP. Equity of access to rehabilitation services in Australia. Wollongong: Centre for Health Service Development, Australian Health Services Research Institute, 2011.

6 Department of Health. Chronic disease management - individual allied health services under Medicare. Provider information. Canberra: Commonwealth of Australia; 2014. http://www.health.gov.au/internet/ main/publishing.nsf/Content/health-medicare-health_pro-gp-pdf-allied-cnt. htm (accessed Mar 2017).

7 Carod-Artal J, Egido JA, González JL, Varela de Seijas E. Quality of life among stroke survivors evaluated 1 year after stroke: experience of a stroke unit. Stroke 2000; 31: 2995-3000.

8 Deloitte Access Economics. The economic impact of stroke in Australia, 2013. Melbourne: National Stroke Foundation; 2013. https://strokefoundation.org. au/What-we-do/Research/Economic-impact-of-stroke-in-Australia (accessed Oct 2016)
9 National Stroke Foundation. National Stroke Audit - Rehabilitation services report 2014. Melbourne: National Stroke Foundation, 2014. https://informme.org.au/en/stroke-data/Rehabilitation-audits (accessed Oct 2016).

10 Shi YZ, Xiang YT, Yang Y, et al. Depression after minor stroke: the association with disability and quality of life - a l-year follow-up study. Int J Geriatr Psychiatry 2016; 31: 421-427.

11 Shi Y, Xiang Y, Yang Y, et al. Depression after minor stroke: prevalence and predictors. J Psychosom Res 2015; 79: 143-147.

12 Hildebrand M, Brewer M, Wolf $\mathrm{T}$. The impact of mild stroke on participation in physical fitness activities. Stroke Res Treat 2012; 2012: 548682.

13 Yu S, Muhunthan J, Lindley R, et al. Driving in stroke survivors aged 18-65 years: the Psychosocial Outcomes in Stroke (POISE) Cohort Study. Int J Stroke 2016; 11: 799-806.

14 Hird MA, Vesely KA, Christie LE, et al. Is it safe to drive after acute mild stroke? A preliminary report. J Neurol Sci 2015; 354: 46-50.

15 Kernan WN, Viscoli CM, Brass LM, et al. The Stroke Prognosis Instrument II (SPI-II): a clinical prediction instrument for patients with transient ischemia and nondisabling ischemic stroke. Stroke 2000; 31: 456-462.

16 Kono Y, Kawajiri H, Kamisaka K, et al. Predictive impact of daily physical activity on new vascular events in patients with mild ischemic stroke. Int J Stroke 2015; 10: 219-223.

17 Hackam DG, Spence JD. Combining multiple approaches for the secondary prevention of vascular events after stroke: a quantitative modeling study. Stroke 2007; 38: 1881-1885. 